\title{
(ADP-ribose) polymerase 1 and AMP-activated protein kinase mediate progressive dopaminergic neuronal degeneration in a mouse model of Parkinson's disease
}

\author{
TW Kim ${ }^{1}$, HM Cho ${ }^{1}$, SY Choi ${ }^{1}$, Y Suguira ${ }^{2}$, T Hayasaka ${ }^{2}$, M Setou ${ }^{2}$, HC Koh ${ }^{3}$, E Mi Hwang ${ }^{4}$, JY Park ${ }^{5}$, SJ Kang ${ }^{6}$, \\ HS Kim ${ }^{1}, \mathrm{H} \mathrm{Kim}^{1}$ and W Sun ${ }^{\star 1}$
}

Genetic and epidemiologic evidence suggests that cellular energy homeostasis is critically associated with Parkinson's disease (PD) pathogenesis. Here we demonstrated that genetic deletion of Poly (ADP-ribose) polymerase 1 completely blocked 6-hydroxydopamine-induced dopaminergic neurodegeneration and related PD-like symptoms. Hyperactivation of PARP-1 depleted ATP pools in dopaminergic (DA) neurons, thereby activating AMP-activated protein kinase (AMPK). Further, blockade of AMPK activation by viral infection with dominant-negative AMPK strongly inhibited DA neuronal atrophy with moderate suppression of nuclear translocation of apoptosis-inhibiting factor (AIF), whereas overactivation of AMPK conversely strengthened the 6-OHDA-induced DA neuronal degeneration. Collectively, these results suggest that manipulation of PARP-1 and AMPK signaling is an effective therapeutic approach to prevent PD-related DA neurodegeneration.

Cell Death and Disease (2013) 4, e919; doi:10.1038/cddis.2013.447; published online 14 November 2013

Subject Category: Neuroscience

Parkinson's disease (PD) is one of the most common debilitating neurodegenerative diseases and is characterized by motor deficits, such as bradykinesia, rest tremor, rigidity, and postural instability. ${ }^{1}$ These symptoms are mainly associated with the slow but progressive loss of dopamine in the striatum, mainly due to the degeneration of dopaminergic (DA) neurons in the substantia nigra (SN). The mechanism underlying the degeneration of DA neurons has been examined in various animal models of PD. ${ }^{2,3}$ One prominent hypothesis is that impaired energy metabolism may underlie slow and progressive neurodegeneration. ${ }^{4,5}$ Several studies have demonstrated that mitochondrial or oxidative defects have important roles in PD. ${ }^{6,7}$ Decreased activity of complex I of the mitochondrial electron transport chain was identified in the SN of PD patients, and 1-methyl-4-phenyl-1,2,3,6-tetrahydropyridine (MPTP), a selective complex I inhibitor, induces DA neuronal death in experimental animal models and in humans. $^{8,9}$ Mutations in genes linked to familial PD such as $\alpha$-synuclein, DJ-1, PINK, parkin, and LRRK2 are directly or indirectly associated with mitochondrial dysfunction. ${ }^{10}$

Notably, poly (ADP-ribose) polymerase-1 (PARP-1) has an important role in the neurotoxin-induced DA neuronal death, and the deletion of $P A R P-1$ gene completely rescued DA neurons from MPTP-induced death. ${ }^{11}$ This DNA repair and protein-modifying enzyme is an abundant nuclear protein selectively activated by DNA breaks and has an important role in cellular defense against oxidative stress. ${ }^{12}$ Because overactivation of PARP-1 rapidly depletes ATP, it has been postulated that PD-related DA neuronal death is caused by necrosis due to energy. ${ }^{13,14}$ However, PARP-1 overactivation can directly promote the AIF release from mitochondria by enhanced formation of PAR polymers, ${ }^{15}$ and energy depletion does not appear to be essential for the execution of PARP-1dependent cell death. ${ }^{16,17}$ Therefore, the importance of PARP-1-induced energy depletion in the neurotoxin-induced DA neuronal degeneration remains to be elucidated. We previously reported that DA neurons underwent caspase-independent, Bax- and apoptosis-inducing factor (AIF)-mediated neuronal death in a 6-hydroxydopamine (6-OHDA)-induced animal model of PD. ${ }^{18}$ Interestingly, although Bax deletion completely prevented nuclear translocation of AIF and DA neuronal death, it failed to prevent 6-OHDA-induced neuronal atrophy. This observation suggests that DA neuronal atrophy is separately controlled by other biochemical mechanisms, independent of Bax-dependent AIF translocation. In the present study, we further demonstrate that PARP-1 promotes both ATP depletion and AIF translocation, and subsequently activates AMP-dependent protein kinase

\footnotetext{
${ }^{1}$ Department of Anatomy, BK21 program, Korea University College of Medicine, 126-1 Anam-Dong, Sungbuk-Gu, Seoul 136-705, Korea; ${ }^{2}$ Department of Cell Biology and Anatomy, Hamamatsu University School of Medicine, 1-20-1 Handayama, Higashi-ku, Hamamatsu, Shizuoka 431-3192, Japan; ${ }^{3}$ Department of Pharmacology, College of Medicine, Hanyang University, Seoul 133-791, Korea; ${ }^{4}$ Center for Functional Connectomics, KIST, Seongbuk-Gu, Seoul 136-791, Korea; ${ }^{5}$ Department of Physiology, Gyeongsang National University School of Medicine, 90 Chilam-Dong, Jinju 660-751, Korea and ${ }^{6}$ Department of Molecular Biology, Sejong University, 98 Gunja-dong, Gwangjin-gu, Seoul 143-747, Korea

${ }^{*}$ Corresponding author: Professor W Sun, Department of Anatomy, Korea University College of Medicine, Seoul 136-705, Korea. Tel: +82 29206404 ; Fax: +82 2929 5696; E-mail: woongsun@korea.ac.kr

Keywords: PARP-1; ATP; AMPK; 6-OHDA; Parkinson's disease

Abbreviations: PARP-1, poly (ADP-ribose) polymerase-1; 6-OHDA, 6-hydroxydopamine; AMPK, AMP-mediated protein kinase; IMS, image mass spectrometry; HPLC, high-performance liquid chromatography; AIF, apoptosis-inducing factor; ATP, adenosine triphosphate; AMP, adenosine monophosphate

Received 07.8.13; revised 11.10.13; accepted 14.10.13; Edited by A Verkhratsky
} 
(AMPK) during 6-OHDA-induced progressive DA neuronal degeneration. Further, functional blockade of PARP-1 or AMPK activation prevents DA neuronal atrophy, suggesting that AMPK is an important regulator of PARP-dependent DA neuronal degeneration and could be an important and novel therapeutic target for PD.

\section{Results}

Effect of 6-OHDA striatal injection on DA neuronal degeneration in wild-type and PARP-1-KO mice. We first explored the extent of DA neuronal atrophy and cell death in WT and PARP-1-KO mice 2 weeks after 6-OHDA injection (Figure 1). TH expression was markedly reduced in ipsilateral DA neurons of WT mice but was largely spared in PARP-1-KO DA neurons (Figures 1a-e). We previously demonstrated that phosphorylation of C-Jun (P-Jun) is a suitable marker for neuronal atrophy (i.e., reduction of $\mathrm{TH}$ expression and cell size). ${ }^{18}$ Following 6-OHDA injection, many $\mathrm{TH}+$ neurons exhibited enhanced P-Jun in WT mice as we previously reported, but the number of P-Jun-labeled cells was significantly reduced in PARP-1-KO mice

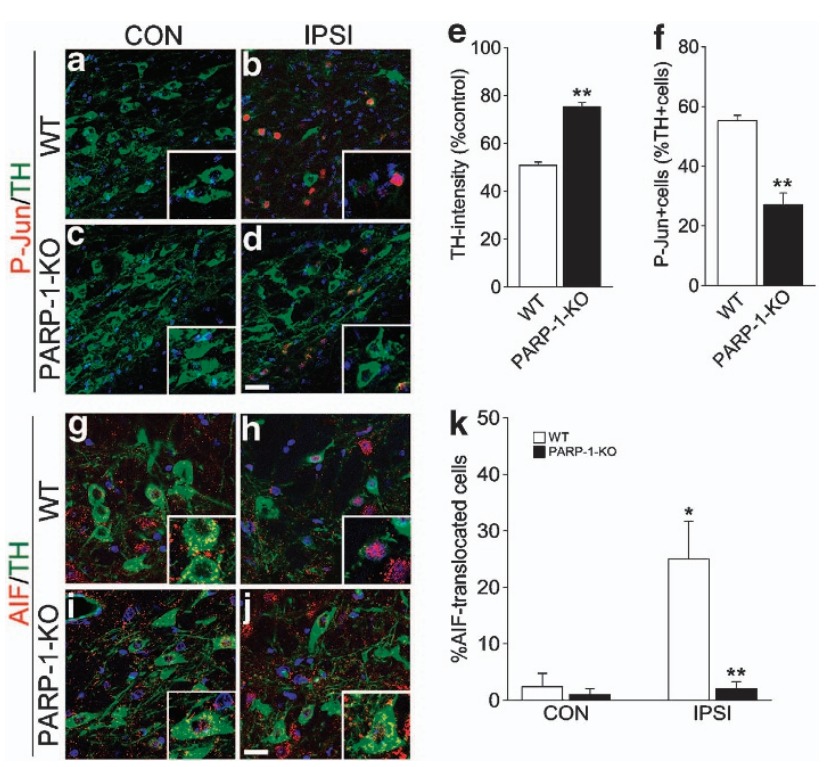

Figure 1 6-OHDA-induced DA neuronal degeneration in WT and PARP-1-KO mice. (a-d) Two weeks after striatal 6-OHDA injection to WT (a, b) or PARP-1-KO (c, d) mice, coronal brain sections containing contralateral (CON; a, c) or ipsilateral (IPSI; b, d) substantia nigra (SN) were immunolabeled with tyrosine hydroxylase $(\mathrm{TH})$ in green and P-Jun in red. Nuclei were counterstained with Hoechst33342 in blue. Insets show magnified images. Scale bar $=20 \mu \mathrm{m}$. (e) Quantification of TH immunoreactivities in the SN of WT and PARP-1-KO mice 2 weeks after 6-OHDA injection. Data are expressed as the percentage mean \pm S.E.M. compared with the mean value of $\mathrm{TH}$ intensity of the CON side in each animal, $n=4$. (f) Percentage of P-Jun-expressing cells among TH + cells in the IPSI side SN of WT and PARP-1KO mice. Data are expressed as the percentage mean \pm S.E.M., $n=4$. $(\mathbf{g}-\mathrm{j})$ Double labeling of TH and AIF in CON $(\mathbf{g}, \mathbf{i})$ and IPSI $(\mathbf{h}, \mathbf{j})$ sides of WT $(\mathbf{g}, \mathbf{h})$ and PARP-1-KO (i, j) mice. Nuclei were counterstained with Hoechst33342 in blue. Insets show large magnification images. (k) Percentage of $\mathrm{TH}+$ cells exhibiting nuclear AIF signals. Data are expressed as the percentage mean \pm S.E.M., $n=4$. ${ }^{\star} P<0.05$, Student's $t$-test comparison with CON versus IPSI sides. ${ }^{\star \star} P<0.05$, Student's t-test comparison with WT versus PARP-1-KO mice
(Figure 1f). In addition, neuronal death was also prevented in PARP-1-KO mice, and DA neurons with nuclear AIF signals were virtually absent in PARP-1-KO mice (Figures 1a-e). Collectively, these results suggest that PARP-1 activation is required for both neuronal atrophy and nuclear translocation of AIF.

Next, we examined whether the absence of AIF translocation and neuronal atrophy in PARP-1-KO mice ultimately affected PD-like phenotypes (Figure 2). Six weeks after 6 -OHDA injection, more than $70 \%$ of DA neurons in the SN were degenerated in WT mice. However, the number of ipsilateral DA neurons was similar to that of the contralateral side in PARP-1-KO mice, indicating that the absence of PARP-1 protected DA neurons against 6-OHDA-induced neurodegeneration (Figures $2 \mathrm{a}-\mathrm{e}$ ). Further, striatal DA nerve fibers were also spared in the PARP-1-KO mice (Figures $2 \mathrm{f}-\mathrm{j}$ ), and the number of apomorphine-induced rotations was reduced in PARP-1-KO mice compared with WT mice, suggesting that DA neurons in PARP-1-KO mice were functional (Figure 2k). Accordingly, higher level of DA contents were detected in the ipsilateral PARP-1-KO striatum compared with the WT (Figure 2l).

Rapid energy depletion of degenerating DA neurons by 6-OHDA in WT mice. PARP-1 hyperactivation induces poly-ADP-ribosylation and subsequent ATP depletion. ${ }^{14,19}$ Therefore, to further evaluate how PARP-1 induces DA neuron degeneration, we assessed the time courses of PARP activation and ATP depletion after 6-OHDA injection. Western blot analysis revealed that protein poly-ADPribosylation occurred as early as 3 days before the signs of DA neuronal atrophy or death and persisted for at least 14 days after 6-OHDA treatment (Figure 3a). Next, we determined the distributions of ATP and AMP in the SN by IMS. Similar to PARP-1 activation, reduced ATP levels in the SN of WT mice were observed from 3 days after 6-OHDA treatment (Figures $3 b-d$ ). However, ATP reduction was completely prevented in PARP-1-KO mice (Figures 3b-d), suggesting that PARP-1 activation critically mediates 6-OHDA-induced ATP depletion in DA neurons.

AMPK phosphorylation is increased in 6-OHDA-injected animal. AMPK is a metabolic fuel gauge that senses changes in the intracellular ATP/AMP ratio. ${ }^{20,21}$ Because we identified a substantial reduction in the ATP/AMP ratio in the SN after 6-OHDA injection, we asked whether AMPK senses ATP depletion and subsequently becomes phosphorylated (Figure 4). Indeed, we found that AMPK phosphorylation was increased at 3 days after 6-OHDA treatment and persisted until 14 days after injection. However, the total protein level was not changed, suggesting that AMP-dependent AMPK phosphorylation occurs mainly at the post-translational level. We also observed reduced levels of phosphorylated ribosomal S6 kinase (pS6K), which is one of the well-known consequences of AMPK activation (Figure 4a). Further, double immunofluorescence labeling revealed that induction of phosphorylated AMPK (Figures 4b-g) and reduction of phosphorylated S6 (Figures 4h-m) were selectively found in degenerating DA neurons in the SN. AMPK phosphorylation by 6-OHDA injection was completely 

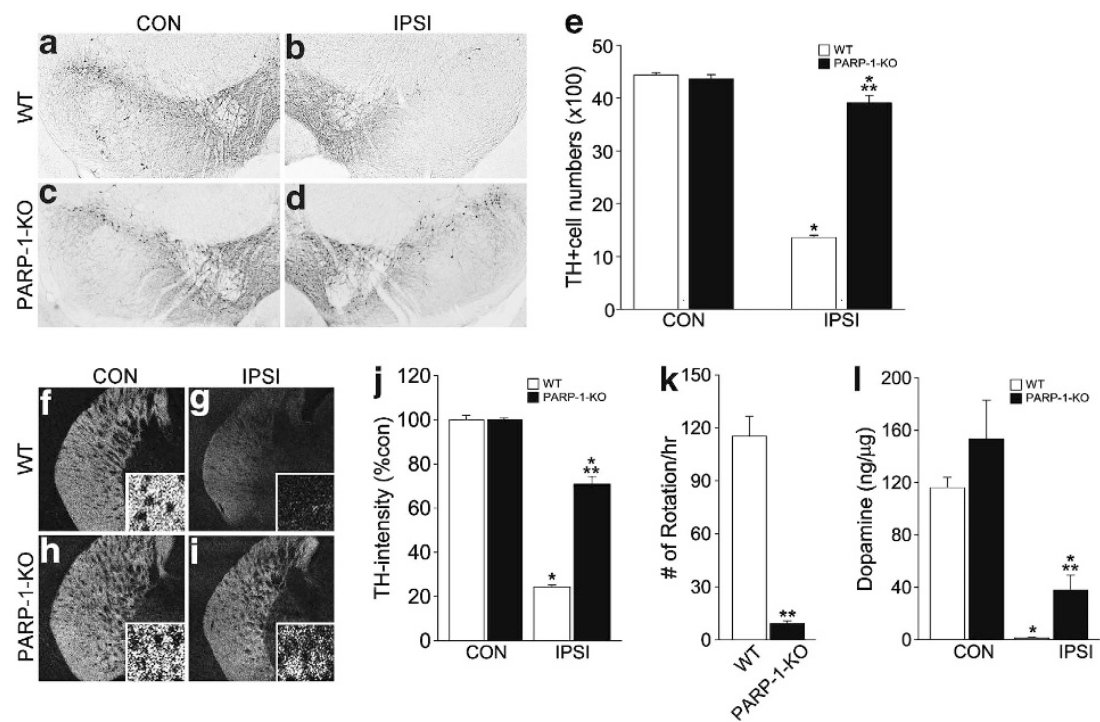

Figure 2 PARP-1-KO mice maintain DA neuronal integrity following 6-OHDA injection. (a-d) TH labeling of CON (a, c) and IPSI (b, d) SN of WT (a, b) and PARP-1-KO (c, d) mice 6 weeks after 6-OHDA injection. (e) Quantification of the TH-expressing DA neurons in the CON and IPSI sides of SN in WT and PARP-1-KO mice. Data are expressed as the percentage mean \pm S.E.M. compared with the mean value of TH intensity of the CON side in each animal, $n=4$. ${ }^{*} P<0.05$, Student's $t$-test comparison with CON versus IPSI sides. ${ }^{* *} P<0.05$, Student's $t$ test comparison with WT versus PARP-1-KO mice. (f-i) Distribution of TH immunoreactive fibers in the CON (f, $\left.\mathbf{h}\right)$ and IPSI ( $\mathbf{g}$, i) sides of WT (f, g) and PARP-1-KO (h, i) mice. (j) Quantification of TH-immunoreactive intensity in the IPSI striatum. Data are expressed as the percentage mean \pm S.E.M. compared with the mean value of TH intensity of CON side in each animal, $n=4$. (k) Number of rotations per hour following apomorphine treatments, $n=6$ for WT and $n=7$ for PARP-1-KO mice. (I) Level of dopamine in the WT and PARP-1-KO striatum, $n=4$

a

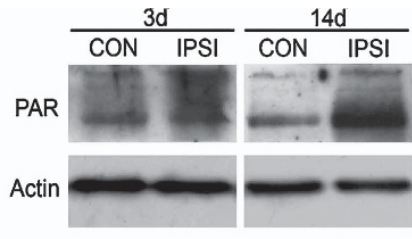

b

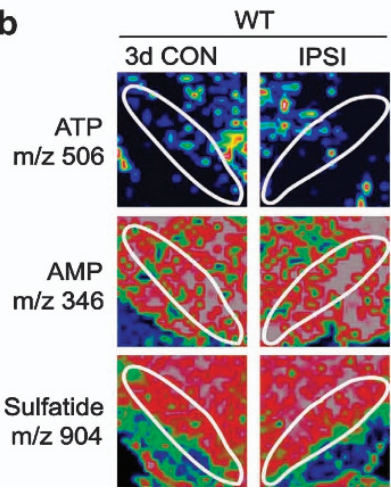

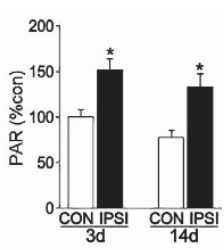
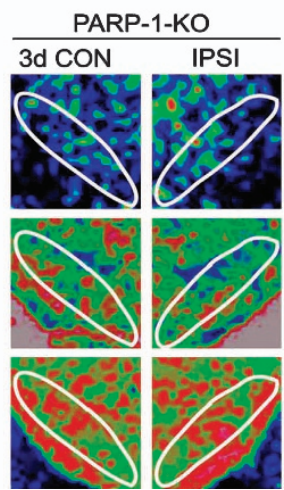
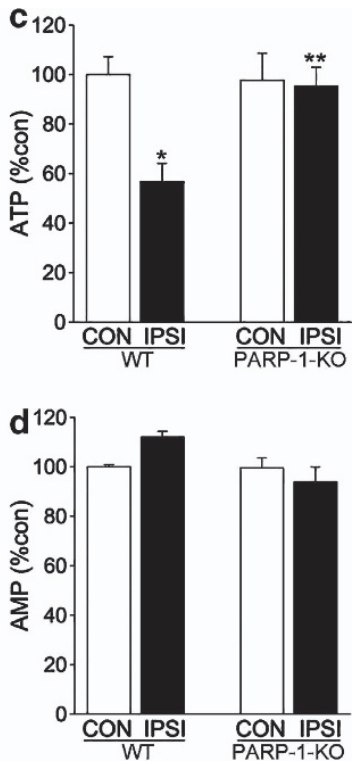

Figure 3 PARP activation and ATP levels in the WT and PARP-1-KO SN following 6-OHDA treatments. (a) Immunoblots of PAR (poly-ADP-ribosylation) in the CON and IPSI sides of SN 3 or 14 days after 6-OHDA injection. Graph shows relative PAR polymer optical densities. $N=3$ (b) ATP and metabolite changes monitored by IMS. At 3 days after 6-OHDA treatment, the midbrain of WT and PARP-1-KO mice was processed, and the contents of ATP (1st) and AMP (2nd) were visualized as pseudocolors. Sulfatide, membrane-rich phospholipids were visualized as a control (last column). (c, d) Quantification of ATP (c), AMP (d), in the CON and IPSI WT and PARP-1-KO SN 3 days after 6-OHDA treatment. SN area was indicated by white line, and the relative intensity of the signals in the SN was obtained using ImageJ software (NIH, Bethesda, MD, USA), $n=4$. ${ }^{*} P<0.05$, Student's $t$-test comparison with CON versus IPSI sides. ${ }^{* *} P<0.05$, Student's $t$-test comparison with WT versus PARP-1-KO mice

blunted in PARP-1-KO mice (Figure 4n), suggesting that PARP-1 triggers AMPK phosphorylation following 6-OHDA treatment.

\section{Acceleration of 6-OHDA-induced DA neuronal degeneration} by metformin. Next, we tested whether additional activation of AMPK affects the extent of the 6-OHDA-induced neuronal degeneration. AMPK activator, metformin, is permeable to brain-blood barrier, ${ }^{22,23}$ and oral administration of metformin $(5 \mathrm{mg} / \mathrm{ml})$ or vehicle (D.W) for 6 days significantly potentiated basal and 6-OHDA-induced AMPK activation (Figure 5a). Thus, we fed metformin in drinking water for 14 days to mice 

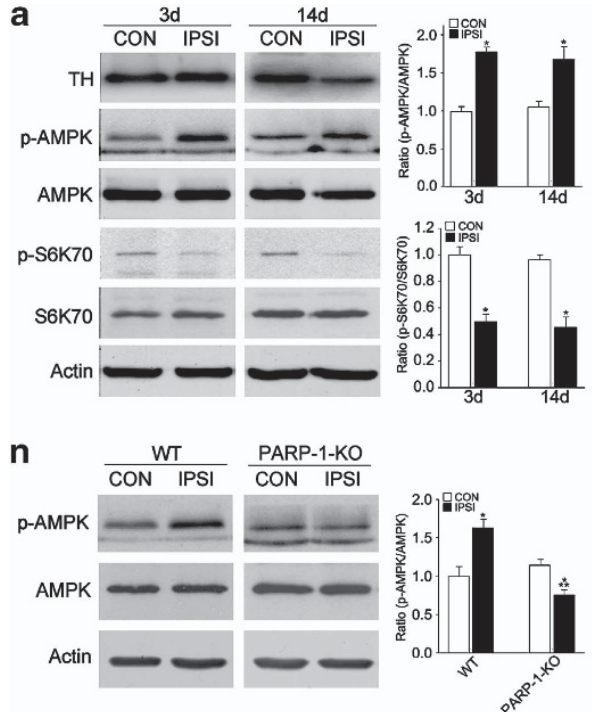
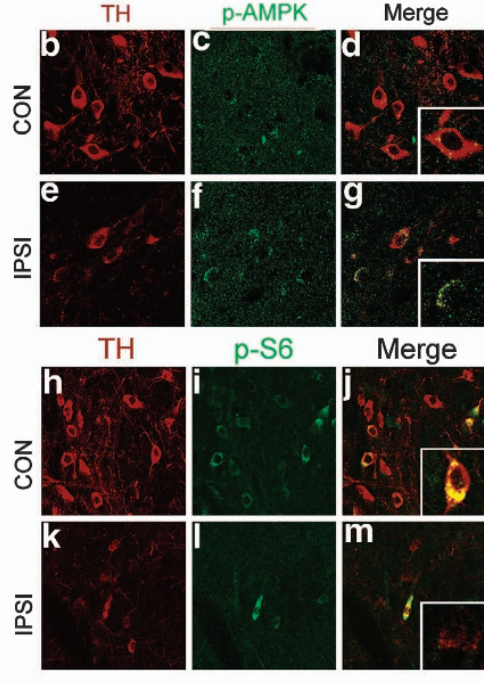

Merge

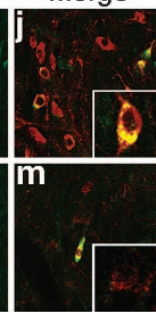

Figure 4 AMPK activation in degenerating DA neurons. (a) At 3 and 14 days after 6-OHDA treatment, SNs from CON and IPSI sides were micropunched, and the expression levels of TH, p-AMPK, AMPK, pS6K70, S6K70, and actin were assessed, $n=3$. The graphs represent the ratios of band densities (p-AMPK/AMPK and ps6K70/ S6K70). (b-g) TH (b, e) and p-AMPK (c, f) were double labeled in the CON (b-d) and IPSI (e-g) SN 14 days after 6-OHDA injection. Merged images are shown in (d, $\mathbf{g})$. Insets show the magnified images. (h-m) TH (h, $\mathbf{k})$ and p-S6 (i, I) were double labeled in the CON (h-j) and IPSI (k-m) SN 14 days after 6-OHDA injection. (n) Expressions of p-AMPK, AMPK, and actin in WT and PARP-1-KO CON and IPSI SN 3 days after 6-OHDA injection, $n=3$. The graph represents the ratios of band densities (p-AMPK/AMPK)
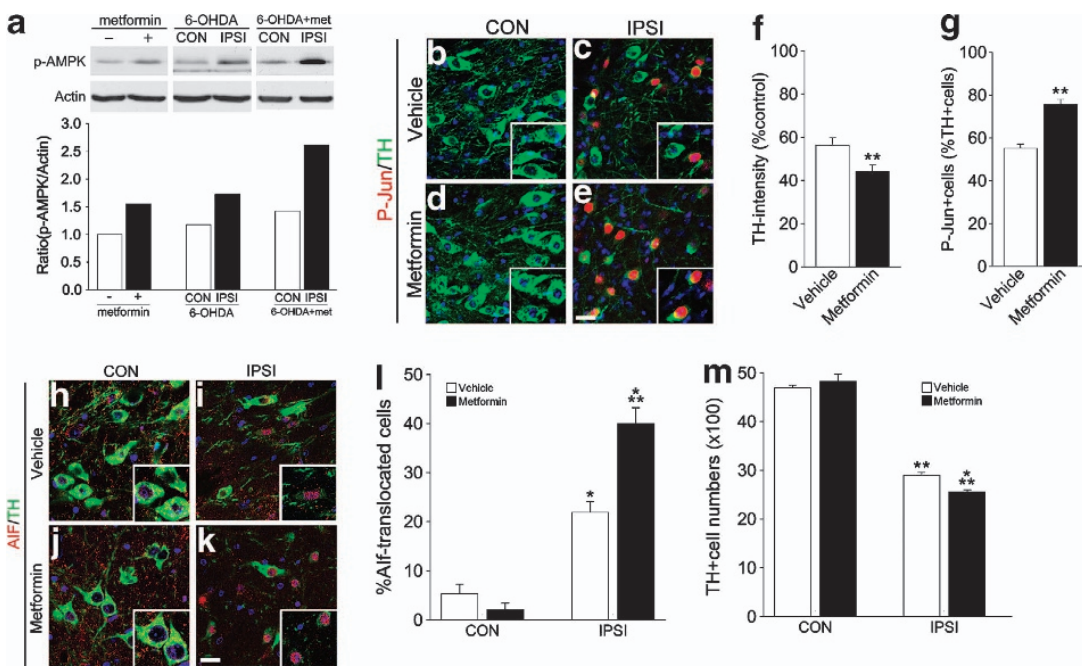

Figure 5 Acceleration of DA neuronal degeneration by metformin (a) Immunoblots of $p$-AMPK, actin in the brain lysates (SN) after receiving metformin in drinking water for 3 days, 3 days after 6-OHDA injection with metformin, or 3 days after 6-OHDA injection without metformin. Graphs at the bottom show relative p-AMPK optical densities. (b-e) Two weeks after striatal 6-OHDA injection to vehicle- $(\mathbf{b}, \mathbf{c})$ or metformin-treated (d, e) mice, coronal brain sections containing contralateral (CON; $\mathbf{b}$, d) or ipsilateral (IPSI; c, e) sides of SN were immunolabeled with TH and P-Jun. Nuclei were counterstained with Hoechst33342. Insets show large magnification images. Scale bar $=20 \mu \mathrm{m}$. (f): Quantification of the intensities of TH immunoreactivity in the SN of vehicle- and metformin-treated mice 2 weeks after 6-OHDA injection. Data are expressed as the percentage mean \pm S.E.M. compared with the mean value of TH intensity of CON side in each animal. $n=4$. (g) Percentage of P-Jun-expressing cells among TH-positive cells in the IPSI side SN of vehicle- and metformin-treated mice. Data are expressed as the percentage mean \pm S.E.M. $n=4$. (h-k): Double labeling of TH and AIF in CON $(\mathbf{h}, \mathbf{j})$ or IPSI (i, k) sides of vehicle- $(\mathbf{h}, \mathbf{i})$ or metformin-treated (j, k) mice. Nuclei were counterstained with Hoechst33342. Insets show large magnification images. (I) Percentage of TH-positive cells exhibiting nuclear AIF signals. (m) Quantification of the TH-expressing DA neurons in the CON and IPSI sides of SN in vehicle- and metformin-treated mice. Data are expressed as the percentage mean \pm S.E.M., $n=5$. ${ }^{*} P<0.05$ in Student's $t$-test comparison with CON versus IPSI sides. ${ }^{* *} P<0.05$ in Student's $t$-test comparison with vehicle- versus metformin-treated mice

with or without striatal 6-OHDA injection. Although metformin treatment alone did not evoke any signs of atrophy or degeneration of neurons, metformin treatment in 6-OHDAinjected mice significantly augmented degenerative changes of DA neurons such as reduction of TH expression and induction of phosphorylation of c-Jun (Figures $5 b-g$ ). Further, translocation of AlF (Figures $5 \mathrm{~h}-\mathrm{l}$ ) was significantly increased, and accordingly the number of surviving DA neurons was significantly reduced (Figure $5 \mathrm{~m}$ ).

Inhibition of DA neuronal degeneration by DN-AMPK infection. To assess the importance of AMPK activation in 
the 6-OHDA-induced DA neuronal degeneration, we examined the effect of AMPK suppression. Adenovirus-coding DN-AMPK that selectively inhibits AMPK activation ${ }^{24}$ was injected into the mouse striatum 3 days before 6-OHDA treatment. The injected adenovirus was retrogradely traced to the SN DA neurons, and we were able to selectively infect $40-60 \%$ of DA neurons in the SN 7 days after viral infection (Figures 6a-f). Further, DN-AMPK infection efficiently blocked 6-OHDA-induced phosphorylation of ACC, which is a downstream event of AMPK activation, indicating the efficient suppression of 6-OHDA-induced AMPK activation (Figure 6g).

By 2 weeks after 6-OHDA treatment, DN-AMPK infection prevented 6-OHDA-induced atrophic changes of DA neurons, such as reduced $\mathrm{TH}$ expression and increased c-Jun phosphorylation, as revealed by immunoblots (Figures $7 \mathrm{a}-\mathrm{c}$ ) and immunohistochemical analysis (Figures 7d-i). In addition, AIF translocation was also significantly attenuated (Figures $7 j-n)$, suggesting that AMPK activation appears to have a significant role in 6-OHDA-induced neuronal degeneration.

We also monitored the long-term effects of transient AMPK blockade. Adenovirus-induced DN-AMPK expression was reduced by 2 weeks and had disappeared 4 weeks after virus infection (data not shown), allowing us to examine the effects of transient suppression of early-phase AMPK induction. We observed a marginal but significant increase in the number of $\mathrm{TH}+\mathrm{DA}$ neurons (Figures $8 \mathrm{a}-\mathrm{e}$ ), spared $\mathrm{TH}+$ fibers in the
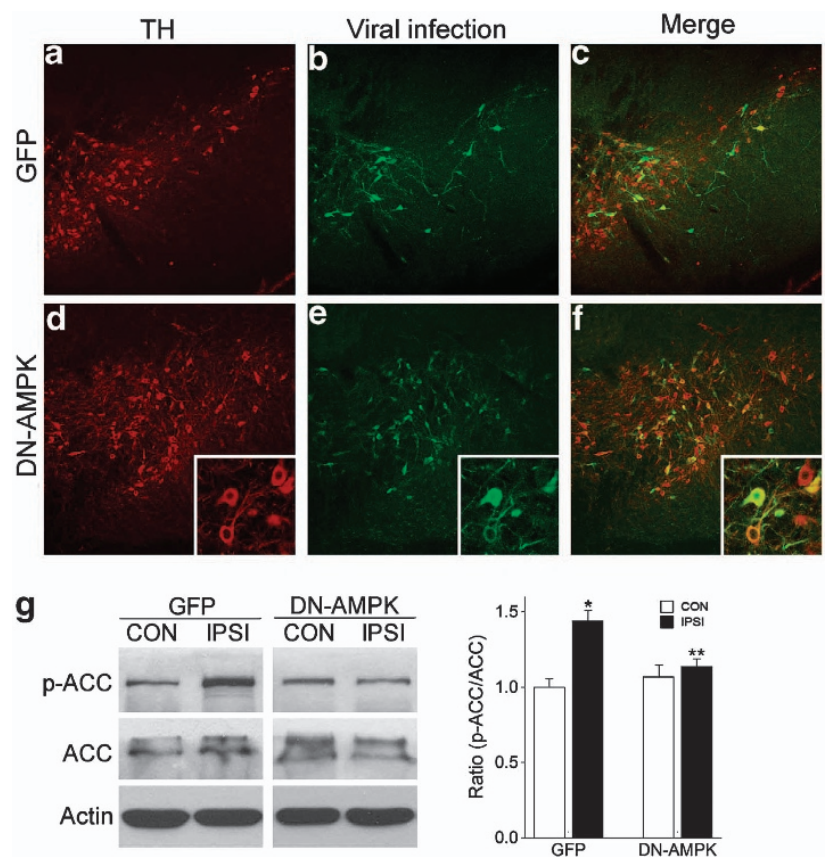

Figure 6 Retrograde labeling and inhibition of AMPK activation in DA neurons by DN-AMPK adenovirus. (a-f) Images taken 2 weeks after injection of GFP (a-c) or DN-AMPK (d-f) virus to adult mouse striatum. TH $(\mathbf{a}, \mathbf{d})$ and GFP $(\mathbf{b}, \mathbf{e})$ were double labeled in the SN 14 days after virus injection. Merged images are shown in (c, f). Insets in ( $\mathbf{d}-\mathbf{f})$ show magnified images of infected DA neurons. (g) Expressions of p-ACC, ACC, and actin in SN 17 days after GFP or DN-AMPK virus injection. The graph represents the ratio of band densities ( $p-A C C / A C C)$ from western blotting experiments, $n=3$ striatum (Figures $8 f-i)$, and reduced rotation behavior following amphetamine injection (Figure 8j). DA levels were slightly increased in the DN-AMPK-infected striatum, but this improvement was not statistically significant (Figure 8k). Collectively, these results suggest that the blockade of increased AMPK activity in the early phase of neuronal atrophy is sufficient to modify subsequent disease progression.

\section{Discussion}

PARP-1 activation is an early event required for 6-OHDAinduced DA neuronal death. In this study, we provided evidence that 6-OHDA-induced DA neuronal degeneration is mediated by PARP-1 activation. PARP-1 is a nuclear enzyme that senses DNA damage and induces DNA repair pathways for cell survival. However, hyperactivation of PARP-1 both depletes cellular ATP and promotes AIF-dependent neuronal death. ${ }^{15,25}$ We previously reported that 6-OHDA-induced DA neuronal death is dependent on Bax gene function and is mediated by AIF activation. ${ }^{18}$ Therefore, both Bax and PARP-1 are appeared to be required for AIF activation. Considering that several reports have demonstrated that PARP-1 is upstream of Bax activation in necrotic cell death pathways, ${ }^{26}$ PARP-1 may promote Bax translocation into mitochondria for subsequent AIF activation. Further, MPTP-induced DA neuronal death, which occurs via caspase-dependent apoptosis, is also both PARP-1- and Bax-dependent, ${ }^{11,27}$ confirming that sequential gene functions (PARP-1 $\rightarrow \mathrm{Bax} \rightarrow \mathrm{AIF}$ ) that mediate DA neuronal death are conserved in many animal models of PD. Although both PARP-1-KO and Bax-KO mice did not show AIF induction or subsequent DA neuronal death, these two animals showed markedly different levels of DA neuron atrophy. Deletion of Bax function failed to prevent 6-OHDAinduced neuronal atrophy, and affected DA neurons exhibited reduced expression of $\mathrm{TH}$ and increased phosphorylation of c-Jun. ${ }^{18}$ Accordingly, Bax-KO mice failed to exhibit a significant improvement of behavioral motor performance. In contrast, surviving PARP-1-KO DA neurons did not show any signs of neuronal atrophy, and these animals showed greatly improved behavioral performance. Therefore, these data suggest that PARP-1 activation is upstream of DA neuronal atrophy as well as cell death, whereas Bax activation only mediates the pathway directly linked to the neuronal death, which is AIF activation. In this respect, neuronal atrophy appears to be mediated by PARP-1 activation, independent of Bax.

AMPK activation is downstream of 6-OHDA-induced PARP-1 activation. PARP-1 activation leads to energy depletion and cell death via a caspase-independent mechanism. ${ }^{28,29}$ Several studies have suggested that energy deficits are associated with neurodegenerative diseases, including $\mathrm{PD}$, and energy metabolism is an important therapeutic target. $^{30,31}$ We hypothesized that PARP-1dependent ATP depletion mediated 6-OHDA-induced DA neuronal atrophy. Direct imaging of ATP levels in the SN after 6-OHDA treatment supported our theory. IMS could minimize the degradation of energy metabolites with rapid metabolic turnover rates, such as ATP and ADP, because 

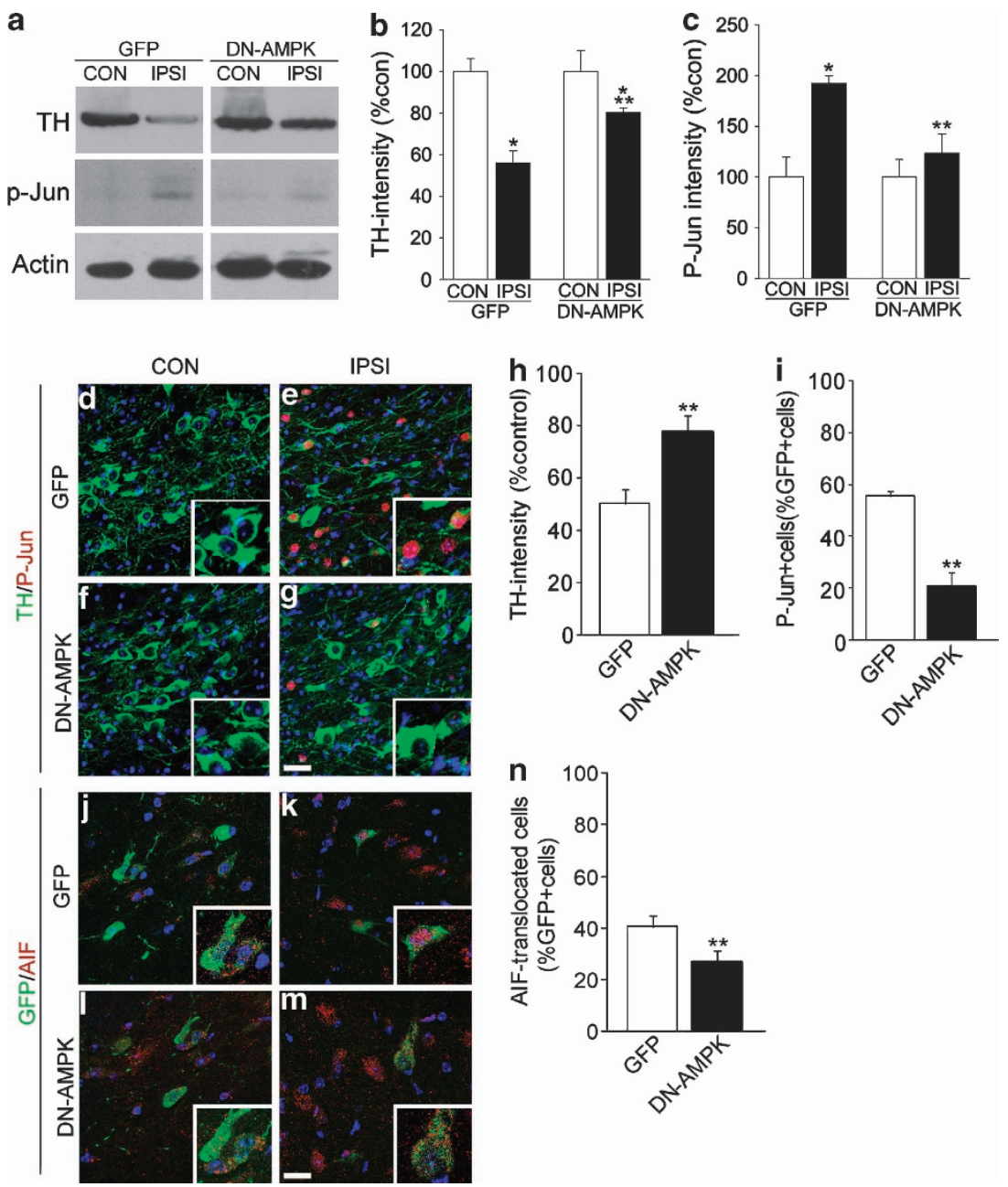

Figure 7 Inhibition of DA neuronal degeneration by DN-AMPK. (a) Immunoblots of TH, P-Jun, and actin in the CON and IPSI sides of GFP or DN-AMPK virus-injected SN 2 weeks after striatal 6-OHDA injection. (b, c) Quantification of the relative optical densities of TH (b) and p-Jun (c). $n=3 ;{ }^{*} P<0.05$, Student's $t$-test comparison with CON versus IPSI; ${ }^{* *} P<0.05$, Student's t-test comparison with GFP versus DN-AMPK virus-injected mice. (d-g) TH (green) and P-Jun (red) immunolabeling in the CON (d, f) and IPSI $(\mathbf{e}, \mathbf{g})$ sides of the SN 2 weeks after 6-OHDA injection with GFP (d, e) or DN-AMPK (f, $\mathbf{g})$ virus. (h-i) Quantification of TH intensity (h) and P-Jun + cells (i) among GFPlabeled cells. Data are expressed as the percentage mean \pm S.E.M., $n=4 .{ }^{*} P<0.05$, Student's $t$-test comparison. (j-m) Double labeling of GFP and AIF in CON (j, I) and IPSI (k, $\mathbf{m})$ sides of GFP (j, $\mathbf{k})$ and DN-AMPK (I, $\mathbf{m})$ virus-injected mice. Nuclei were counterstained with Hoechst33342 in blue. Insets show magnified images. (n) Percentage of $\mathrm{TH}+$ cells exhibiting nuclear AIF signals. Data are expressed as the percentage mean \pm S.E.M., $n=4 .{ }^{*} P<0.05$, Student's $t$-test comparison

the matrix solvent applied to the tissue contains an organic solution that suppresses most postmortem enzymatic activities by denaturing the enzymes. ${ }^{32}$ Using this imaging technique, we found that ATP depletion occurred rapidly in the first 3 days after 6-OHDA injection, which is before DA neuronal atrophy and death. Thus, the data imply that ATP depletion is an early event of DA neuronal degeneration. We also found that rapid ATP depletion is dependent on the PARP-1 hyperactivation because PARP-1-KO completely prevented this event. In response to PARP-1 activation and ATP depletion, AMPK senses ATP depletion and initiates catabolic responses. Accordingly, AMPK phosphorylation was also absent in PARP-1-KO mice at 3 days after 6-OHDA treatment, further confirming that PARP-1 activation is required for ATP depletion and AMPK activation.

AMPK activation mediates DA neuronal atrophy. In this study, we found that AMPK activation is necessary for the DA neuronal atrophy. A schematic illustration of the proposed molecular pathways underlying 6-OHDA-induced DA neurodegeneration is shown in Figure 9. Overactivation of AMPK by co-treatment with metformin accelerated DA neurodegeneration. Conversely, blockade of early AMPK induction significantly inhibited 6-OHDA-induced DA neuronal atrophy and moderately repressed the nuclear translocation of AIF. AMPK is a Ser/Thr kinase that has a fundamental regulatory role in energy homeostasis, acting as a sensor of energy balance; it is phosphorylated and activated when energy levels are low. ${ }^{33,34}$ AMPK is expressed in most mammalian tissues and cell types, including neurons. ${ }^{35,36}$ Considering that PARP-1-dependent AMPK activation is required for the DA neuronal atrophy, which is not prevented by Bax deletion, ${ }^{18}$ it appears that AMPK activation is linked with PARP-1-dependent neuronal atrophy. AMPK is a metabolic sensor that mediates important metabolic processes. For example, pharmacological activation of AMPK 

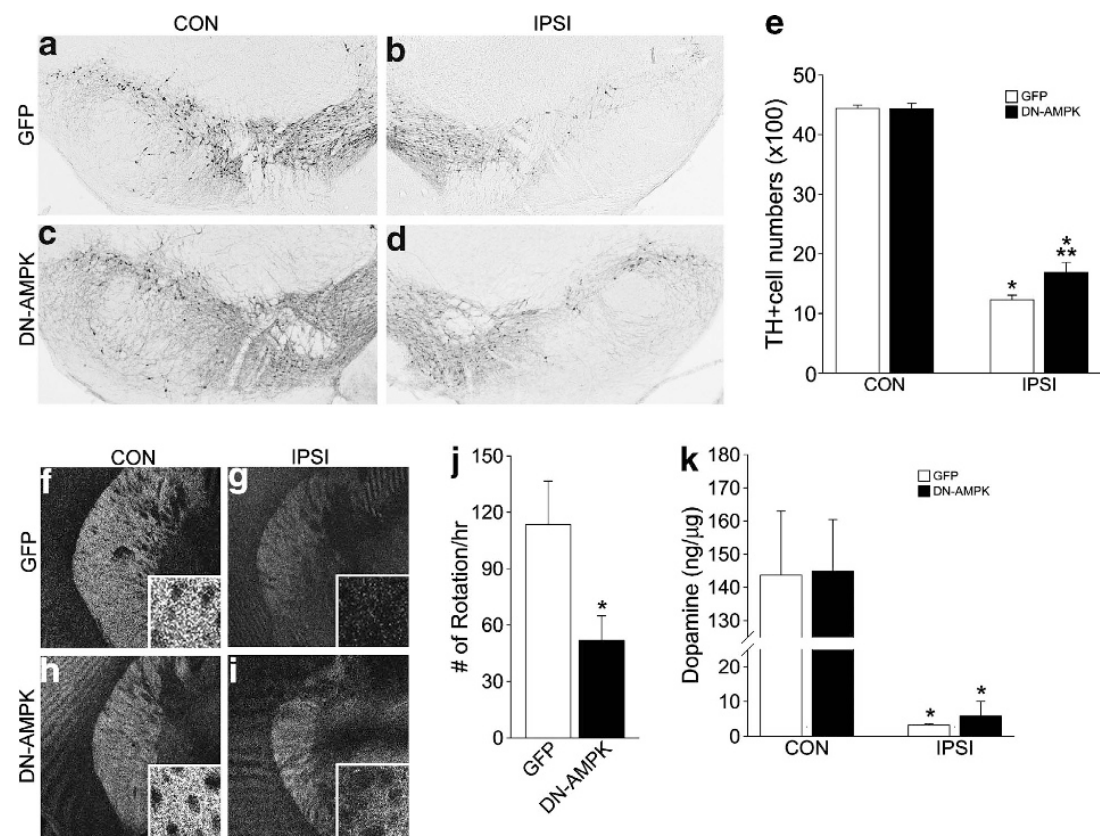

Figure 8 Long-term effect of DN-AMPK on DA neuronal degeneration. (a-d) TH labeling of CON (a, $\mathbf{c}$ ) and IPSI (b, d) SN of GFP (a, b) and DN-AMPK virus-injected mice (c, d) 6 weeks after 6-OHDA injection. (e) Quantification of TH-expressing DA neurons in the CON and IPSI sides of SN in GFP and DN-AMPK virus-injected mice. Data are expressed as \%mean \pm S.E.M compared with the mean value of TH intensity of the CON side in the same animal, $n=4$. ${ }^{*} P<0.05$, Student's $t$-test comparison with CON versus IPSI sides; ${ }^{* *} P<0.05$, Student's $t$-test comparison with GFP versus DN-AMPK mice. (f-i) Distribution of TH immunoreactive fibers in the $\operatorname{CON}(\mathbf{f}, \mathbf{h})$ and IPSI $(\mathbf{g}, \mathbf{i})$ sides of GFP $(\mathbf{f}, \mathbf{g})$ and DN-AMPK virus-injected mice $(\mathbf{h}, \mathbf{i})$. (j) Number of rotations per hour following apomorphine treatments, $n=6$ for GFP and $n=7$ for DN-AMPK mice. ${ }^{*} P<0.05$, Student's $t$-test comparison with CON versus IPSI sides. (k) Striatal DA levels in GFP and DN-AMPK virus-injected mice, $n=5$

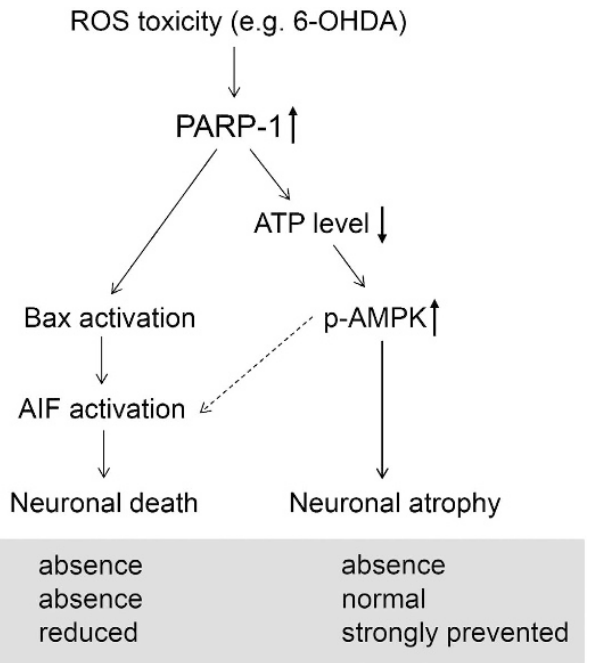

Figure 9 Schematic diagram for the proposed signaling pathways of 6OHDA-induced DA neurodegeneration. ROS toxicity induced by 6-OHDA treatments promotes the hyperactivation of PARP-1 in the DA neurons, which in turn promotes a rapid depletion of ATP and mitochondrial Bax translocation. Reduced cellular ATP level triggers AMPK activation, and activated AMPK initiates neuronal atrophy. In addition, AMPK activation appears to affect the nuclear translocation of AIF and subsequent DA neuronal death. Therefore, blockade of PARP-1 activity completely prevents DA neuronal atrophy and death, whereas Bax knockout only prevents neuronal death. ${ }^{18}$ In contrast, suppression of AMPK strongly inhibits neuronal atrophy but partly reduces AIF-dependent neuronal death inhibits protein synthesis via the modification of mammalian target of rapamycin (mTOR) pathways. ${ }^{37-40}$ Therefore, neuronal atrophy and reduction of cell size are likely associated with a hyperactivation of AMPK and subsequent suppression of protein synsthesis. Interestingly, recent studies showed that prevention of PTEN-mTOR signaling can trigger neuronal regeneration, and viral activation of this signaling can prevent 6-OHDA-induced DA neuronal degeneration. ${ }^{41,42}$ Therefore, reduced PTEN-mTOR signaling followed by AMPK inhibition may relieve Parkinson's symptoms caused by the functional insufficiency of DA neurons.

Pharmacological activation of AMPK also induces autophagy, ${ }^{43-45}$ and autophagy is promoted by AMPK through mammalian autophagy-inducing kinase Ulk1 under starvation conditions. ${ }^{46}$ Further, mutations in genes mediating autophagic pathways, such as PARKIN and PINK, are closely associated with PD. ${ }^{47,48}$ Although we failed to observe the changes in the level of autophagy-related proteins (i.e., no activation of LC3-II and Beclin 1) following 6-OHDA injection in our mouse model, ${ }^{18}$ these results suggest that changes in the metabolic signals could be common events in sporadic and genetic PD.

Although the inhibition of AMPK activation primarily prevented DA neuronal atrophy, it also moderately reduced nuclear translocation of AIF and subsequent neuronal death, suggesting that there is a cross talk between AMPK-dependent neuronal atrophy and Bax-AIF-dependent 
neuronal death pathways. Although we did not directly address this issue, it is known that prolonged AMPK activation increases the expression of $\mathrm{BH} 3-$ only protein Bim following energy depletion. ${ }^{49}$ Because Bim is a direct activator of Bax, ${ }^{50}$ the activation of BH3-only proteins by AMPK hyperactivation may transmit the atrophic signals to the cell death cascade.

AMPK activation has commonly found in many neurological diseases, including stroke, Huntington's disease (HD), and Alzheimer's disease (AD). ${ }^{51-53}$ It is generally accepted that AMPK activation can worsen neurodegenerative process. AMPK overactivation facilitated brain atrophy via increased formation of $\mathrm{Htt}$ aggregates in a mouse animal model of HD. ${ }^{54}$ In addition, activation of AMPK by metformin or AICAR triggered dendritic spine loss (a hallmark of atrophy) in mature hippocampal neurons in an AD model. ${ }^{55}$ These results suggest that overactivation of AMPK can contribute to the neuronal atrophy and subsequent neuronal degeneration in many different neurodegenerative diseases including PD.

\begin{abstract}
Materials and Methods
Animals and surgery. PARP-1-KO mice were obtained from Jackson Laboratory (Bar Harbor, ME, USA) and maintained by inbreeding with mice on a $\mathrm{C} 57 \mathrm{~B} / / 6$ background. Homozygous mice were obtained by breeding heterozygous male and female mice, and the genotypes of the offspring were individually assessed by polymerase chain reaction (PCR) as previously described. ${ }^{56}$ For stereotaxic injection of 6-OHDA, adult male mice (25-30 g) were deeply anesthetized with sodium pentobarbital $(50 \mathrm{mg} / \mathrm{kg})$ and placed in a stereotaxic device (Stoelting, Wood Dale, IL, USA), and 6-OHDA (15 $\mu \mathrm{g}$ in $1.5 \mu \mathrm{l}$ of phosphate-buffered saline (PBS) containing $0.02 \%$ ascorbate) was injected into the right striatum (anterior, $+0.05 \mathrm{~mm}$; medial, $-0.2 \mathrm{~mm}$; dorsal, $-0.35 \mathrm{~mm}$ relative to bregma) by a 30-gauge microsyringe at a rate of $0.5 \mu / / \mathrm{min}$. After a 15-min pause, the needle was slowly withdrawn.
\end{abstract}

Virus preparation and infection. Adenovirus carrying dominant-negative mutant AMPK $\alpha 2$ cDNA (DN-AMPK; kindly provided by Dr. Joohun Ha, Kyung Hee University School of Medicine, Seoul, Korea) was prepared using a ViraPower adenovirus expression system (Invitrogen, Carlsbad, CA, USA). AMPK $\alpha 2$ was PCR-amplified and subcloned into a pAd/CMV/V5-DEST vector (Invitrogen) using a Gateway system with LR clonase (Invitrogen). eGFP cDNA was used as a control after subcloning into a pAd/CMV/V5-DEST vector. Infected 293 cells from 25 to $5015-\mathrm{cm}$ tissue culture plates were collected and centrifuged in a Beckman CS-6R desktop centrifuge (Brea, CA, USA) at 1500 r.p.m. for $5 \mathrm{~min}$. The cells were resuspended in the culture medium without fetal bovine serum and were lysed by three freeze-thaw cycles. Cell lysates were clarified to remove cellular debris by centrifugation in a Beckman CS-6R at 3000 r.p.m. for 10 min. For stereotaxic injection, virus particles were concentrated, and $1 \times 10^{12}$ particles $/ \mathrm{ml}$ and $1.5 \mu \mathrm{l}$ viral solution were injected into the right striatum (anterior, $+0.06 \mathrm{~mm}$; medial, $-0.22 \mathrm{~mm}$; dorsal, $-0.35 \mathrm{~mm}$ relative to bregma) with a 30 -gauge microsyringe at a rate of $0.5 \mu \mathrm{l} / \mathrm{min}$.

Histology. For immunohistochemical analysis, mice were perfused with $4 \%$ paraformaldehyde, and the brains were isolated. Following post fixation in the same fixative overnight, brains were cryoprotected in $30 \%$ sucrose, sectioned serially $(40 \mu \mathrm{m})$, and stored in $50 \%$ glycerol/50\% PBS solution at $-20^{\circ} \mathrm{C}$ until use. Every 6th or 12th section containing the SN was blocked with $3 \%$ bovine serum albumin (BSA) in PBS for 30 min. Primary antibodies were applied to the sections overnight at room temperature. Primary antibodies used in this study were rabbit polyclonal tyrosine hydroxylase (TH; 1:1000; Millipore, Billerica, MA, USA), mouse monoclonal TH (1:500; Sigma, St Louis, MO, USA), rabbit polyclonal phosphorylated-c-Jun $(1: 250$; Cell Signaling, Danvers, MA, USA), rabbit monoclonal AIF (1:1000; Epitomics, Burlingame, CA, USA), goat polyclonal GFP (1: 1000; Abcam, Cambridge, MA, USA), and rabbit monoclonal phospho-S6 Ribosomal Protein (Ser235.236; 91B2; 1:500; Cell Signaling). After several washes with PBS, sections were incubated with appropriate secondary antibodies for $30 \mathrm{~min}$. Nuclei were counterstained with Hoechst33342, and the images were captured with a Zeiss LSM510 confocal microscope (Oberkochen, Germany).
For the measurement of the intensity of $\mathrm{TH}$ immunoreactivity, all images were captured under identical conditions, and staining intensity within cell bodies or in the striatum was obtained using the ImageJ program (http://rsbweb.nih.gov/ij).

Stereological analysis. To quantify the number of TH-positive $(\mathrm{TH}+)$ neurons, an optical dissector method of stereological analysis was used (Kim et al., 2007). Using Stereolnvestigator Software (MicroBrightField, Willston, VT, USA), a fractionator probe was established for each section, and every 6th section covering the entire SN was examined. The number of $\mathrm{TH}+$ neurons in each counting frame was determined by focusing down through the section using a $\times 40$ objective. Our criteria for counting an individual $\mathrm{TH}+$ neuron was the presence of its nucleus within the counting frame or touching the right or top lines but not touching the left or bottom lines. The total number of $\mathrm{TH}+$ neurons in each section of SN was mathematically calculated using the Stereo Investigator program.

Image mass spectrometry (IMS). Tissue blocks were sectioned at $-16^{\circ} \mathrm{C}$ using a cryostat (CM 3050; Leica, Wetzlar, Germany) to a thickness of $5 \mu \mathrm{m}$ as previously described ${ }^{57}$ Although brain blocks were held by an optimum cutting temperature polymer, they were not fully embedded because it was thought that any residual polymer on the tissue slices might degrade the mass spectra. ${ }^{58}$ The frozen sections were thaw-mounted on indium-tin-oxide-coated glass slides (Bruker Daltonics, Billerica, MA, USA). Tissue preparation and matrix-assisted laser desorption/ionization (MALDI) IMS were performed using 9-aminoacridine as the matrix (10 mg/ml, dissolved in $70 \%$ methanol). Matrices were simultaneously applied to the tissue sections to maintain consistent analyte extraction and co-crystallization conditions. MALDI-IMS imaging was performed using an Ultra Flex 2 MALDI-time-of-flight mass spectrometer (Bruker Daltonics) equipped with a $\mathrm{Nd}$ :YAG laser. Data were acquired in the negative reflectron mode. Each spectrum was the result of 100 laser shots at each data point. In this analysis, signals between $\mathrm{m} / \mathrm{z} 100$ and 1000 were collected. The interval between data points was $50 \mu \mathrm{m}$; in total, we obtained $\sim 6000$ data points for each SN. Image reconstruction was performed using Flexlmaging 2.0 (Bruker Daltonics).

Western blotting. For acquisition of $\mathrm{SN}$ tissue, each brain was placed on a cryostat, and coronal slices $(0.5 \mathrm{~mm})$ containing the entire SN were taken. The slices were placed anterior surface up on a slide glass, and the SN was selectively punched out on each brain side with a 1.0-mm micro-punch. Tissues were immediately frozen on dry ice and stored at $-80^{\circ} \mathrm{C}$. Frozen tissue was homogenized with a Tomy handy sonicator (Tomy Seiko, Tokyo, Japan) in lysis buffer ( $50 \mathrm{mM}$ Tris-Cl (pH 7.4), $150 \mathrm{mM} \mathrm{NaCl}, 10 \%$ glycerol, $1 \%$ Triton-X100, $1 \mathrm{mM}$ EDTA, $25 \mathrm{mM} \mathrm{NaF}, 1 \mathrm{mM} \mathrm{Na}_{3} \mathrm{VO}_{4}, 1 \mathrm{mM}$ phenylmethylsulfonyl fluoride, protease inhibitor cocktail). Cell lysates were size-separated through denaturing sodium dodecyl sulfate-polyacrylamide gel electrophoresis. An equal amount of protein for each sample was heated at $100^{\circ} \mathrm{C}$ for $5 \mathrm{~min}$ with an equivalent volume of sample buffer (containing 5\% sodium dodecyl sulfate and $10 \%$ $\beta$-mercaptoethanol) and loaded onto polyacrylamide gels. The proteins were electrotransferred to nitrocellulose membranes in Tris-glycine-methanol buffer. The membrane was blocked for $1 \mathrm{~h}$ at room temperature in a blocking solution mixture of $5 \%$ nonfat dry milk, $0.1 \%$ Tween 20 , and Tris-buffered saline, pH 7.4. The membrane was incubated at $4{ }^{\circ} \mathrm{C}$ overnight with a primary antibody in blocking solution. Primary antibodies used were rabbit polyclonal anti-acetyl-CoA carboxylase (ACC; 1: 1000, Millipore), rabbit polyclonal phospho-AMPK (Thr ${ }^{172}$; $1: 1000$, Millipore), rabbit polyclonal phospho-ACC (Ser ${ }^{79} ; 1: 1000$, Cell Signaling), rabbit polyclonal anti-AMPK (1:1000, Abcam), rabbit polyclonal anti-Poly(ADPRibose) (1:1000; BD Pharmingen, San Jose, CA, USA), rabbit polyclonal phosphor-S6k (1:1000, Cell Signaling), rabbit polyclonal anti-S6k (1:1000, Cell Signaling), rabbit polyclonal phosphorylated-c-Jun (1:1000, Cell Signaling), and mouse monoclonal $\beta$-actin $(1: 5000$, Sigma). Membranes were rinsed with blocking solution three times for $10 \mathrm{~min}$ and incubated with appropriate peroxidase-labeled secondary antibody for $1 \mathrm{~h}$ at room temperature. Blots were washed, and the signal was detected using a Supersignal ChemiLuminiscent detection kit (Pierce/Thermo Fisher Scientific, Waltham, MA, USA) according to the manufacturer's instructions.

Behavioral analysis. For rotation tests, mice were received intraperitoneal injections of $0.5 \mathrm{mg} / \mathrm{kg}$ apomorphine 6 weeks after 6-OHDA injection. Immediately after injection of apomorphine, the animals were individually placed in a test bowl, and the number of clockwise rotations was monitored for $60 \mathrm{~min}^{59}$ 
High-Performance Liquid Chromatography. The levels of DA in the brain tissues were determined using a modified method. ${ }^{60}$ The tissue was homogenized with a Tomy handy sonicator (Tomy Seiko, Tokyo, Japan) in $280 \mu \mathrm{l}$ of $0.2 \mathrm{M}$ perchloric acid containing $0.1 \mathrm{mM}$ EDTA and $20 \mu \mathrm{l}$ of $10 \mu \mathrm{M}$ 3,4-dihydroxybenzylamine hydrobromide as an internal standard. Cell membranes were disrupted using a sonicator (Tomy Seiko) in an ice bath and centrifuged at 13000 r.p.m. for $10 \mathrm{~min}$ at $4^{\circ} \mathrm{C}$. The supernatants were filtered through centrifugal filter devices (Microcon YM-10, Millipore) by centrifuging at 13000 r.p.m. for $15 \mathrm{~min}$ at $4{ }^{\circ} \mathrm{C}$. After filtration, $20 \mu \mathrm{l}$ of the sample was injected directly into an injector (7725i, Rheodyne, Cotati, CA, USA) and analyzed using high-performance liquid chromatography (HPLC) with electrochemical detection. The pellets were used for protein concentration measurements. The HPLC system consisted of an electrochemical detector (ECD-300, EICOM, Kyoto, Japan), a solvent delivery system (515 HPLC-pump; Waters Co., Milford, MA, USA), a column oven (Waters Co.), and a data processor dsCHROM-net (Donam Int., Seoul, Korea). The separation column was a reverse-phase C18 column $(3.0 \mathrm{~mm}$ i.d. $\times 150 \mathrm{~mm}$ EICOMPAK SC-50DS; EICOM), and the guard column was a PREPAK $(4.0 \mathrm{~mm}$ i.d. $\times 5 \mathrm{~mm}$, EICOM). The appendance potential of ECD-300 (carbon electrode versus. $\mathrm{Ag} / \mathrm{AgCl}$ reference electrode) was set at $+750 \mathrm{mV}$. The mobile phase consisted of $90 \mathrm{mM}$ sodium acetate: $100 \mathrm{mM}$ citric acid buffer ( $\mathrm{pH} 3.5$ ) in methanol (83:17, v/v) containing $190 \mathrm{mg} / \mathrm{l}$ sodium-1-octanesulfonic acid and $5 \mathrm{mg} / \mathrm{l}$ $2 \mathrm{Na}$-EDTA. The flow rate was set at $0.5 \mathrm{ml} / \mathrm{min}$ at $30^{\circ} \mathrm{C}$. 3,4-Dihydroxybenzylamine hydrobromide was used as the internal standard for the quantification of DA concentrations. DA levels were calculated using a DA standard (3-hydroxydopamine, dopaminehydrochloride) and normalized to the sample protein levels. DA levels were expressed as $\mathrm{ng} / \mathrm{mg}$ protein.

\section{Conflict of Interest}

The authors declare no conflict of interest.

Acknowledgements. We thank Dr. Seong-Woon Yu and Dr. Han Seok Ko for critical review and thoughtful comments. This research was supported by the Brain Research Program through the National Research Foundation of Korea (NRF) funded by the Ministry of Science, ICT and Future Planning (NRF-2012M3A9C6049933, NRF-2011-0019212, and NRF-2010-0020237).

1. Dawson TM. New animal models for Parkinson's disease. Cell 2000; 101: 115-118.

2. Turmel H, Hartmann A, Parain K, Douhou A, Srinivasan A, Agid $Y$ et al. Caspase-3 activation in 1-methyl-4-phenyl-1,2,3,6-tetrahydropyridine (MPTP)-treated mice. Mov Disord 2001; 16: 185-189.

3. Mladenovic A, Perovic M, Raicevic N, Kanazir S, Rakic L, Ruzdijic S. 6-Hydroxydopamine increases the level of TNFalpha and bax mRNA in the striatum and induces apoptosis of dopaminergic neurons in hemiparkinsonian rats. Brain Res 2004; 996: 237-245.

4. Yang L, Calingasan NY, Wille EJ, Cormier K, Smith K, Ferrante RJ et al. Combination therapy with coenzyme Q10 and creatine produces additive neuroprotective effects in models of Parkinson's and Huntington's diseases. J Neurochem 2009; 109 1427-1439.

5. Ferreira IL, Resende R, Ferreiro E, Rego AC, Pereira CF. Multiple defects in energy metabolism in Alzheimer's disease. Curr Drug Targets 2010; 11: 1193-1206.

6. Schapira AH, Gu M, Taanman JW, Tabrizi SJ, Seaton T, Cleeter M et al. Mitochondria in the etiology and pathogenesis of Parkinson's disease. Ann Neurol 1998; 44: S89-S98.

7. Jenner $\mathrm{P}$, Olanow CW. The pathogenesis of cell death in Parkinson's disease. Neurology 2006; 66: S24-S36.

8. Bloem BR, Irwin I, Buruma OJ, Haan J, Roos RA, Tetrud JW et al. The MPTP model: versatile contributions to the treatment of idiopathic Parkinson's disease. J Neurol Sci 1990; 97: 273-293.

9. Hattori N, Tanaka M, Ozawa T, Mizuno Y. Immunohistochemical studies on complexes I, II, III, and IV of mitochondria in Parkinson's disease. Ann Neurol 1991; 30: 563-571.

10. Schon EA, Przedborski S. Mitochondria: the next (neurode)generation. Neuron 2011; 70: 1033-1053.

11. Mandir AS, Przedborski S, Jackson-Lewis V, Wang ZQ, Simbulan-Rosenthal CM, Smulson $\mathrm{ME}$ et al. Poly(ADP-ribose) polymerase activation mediates 1-methyl-4-phenyl-1, 2,3, 6-tetrahydropyridine (MPTP)-induced parkinsonism. Proc Natl Acad Sci USA 1999; 96 5774-5779.

12. Virag $L$, Szabo $C$. The therapeutic potential of poly(ADP-ribose) polymerase inhibitors Pharmacol Rev 2002; 54: 375-429.

13. Virag L, Salzman AL, Szabo C. Poly(ADP-ribose) synthetase activation mediates mitochondrial injury during oxidant-induced cell death. J Immunol 1998; 161 3753-3759.
14. Los M, Mozoluk M, Ferrari D, Stepczynska A, Stroh C, Renz A et al. Activation and caspase-mediated inhibition of PARP: a molecular switch between fibroblast necrosis and apoptosis in death receptor signaling. Mol Biol Cell 2002; 13: 978-988.

15. Yu SW, Andrabi SA, Wang H, Kim NS, Poirier GG, Dawson TM et al. Apoptosis-inducing factor mediates poly(ADP-ribose) (PAR) polymer-induced cell death. Proc Natl Acad Sci USA 2006; 103: 18314-18319.

16. Tapodi A, Debreceni B, Hanto K, Bognar Z, Wittmann I, Gallyas F Jr. et al. Pivotal role of Akt activation in mitochondrial protection and cell survival by poly(ADP-ribose)polymerase1 inhibition in oxidative stress. J Biol Chem 2005; 280: 35767-35775.

17. Wang Y, Kim NS, Haince JF, Kang HC, David KK, Andrabi SA et al. Poly(ADP-ribose) (PAR) binding to apoptosis-inducing factor is critical for PAR polymerase-1-dependent cell death (parthanatos). Sci Signal 2011; 4: ra20.

18. Kim TW, Moon Y, Kim K, Lee JE, Koh HC, Rhyu IJ et al. Dissociation of progressive dopaminergic neuronal death and behavioral impairments by bax deletion in a mouse model of Parkinson's diseases. PLOS ONE 2011; 6: e25346.

19. Du L, Zhang X, Han YY, Burke NA, Kochanek PM, Watkins SC et al. Intra-mitochondrial poly(ADP-ribosylation) contributes to NAD + depletion and cell death induced by oxidative stress. J Biol Chem 2003; 278: 18426-18433.

20. Woods A, Azzout-Marniche D, Foretz M, Stein SC, Lemarchand P, Ferre $P$ et al. Characterization of the role of AMP-activated protein kinase in the regulation of glucoseactivated gene expression using constitutively active and dominant negative forms of the kinase. Mol Cell Biol 2000; 20: 6704-6711.

21. Carling D, Mayer FV, Sanders MJ, Gamblin SJ. AMP-activated protein kinase: nature's energy sensor. Nat Chem Biol 2011; 7: 512-518.

22. Ma TC, Buescher JL, Oatis B, Funk JA, Nash AJ, Carrier RL et al. Metformin therapy in a transgenic mouse model of Huntington's disease. Neurosci Lett 2007; 411: 98-103.

23. Chen Y, Zhou K, Wang R, Liu Y, Kwak YD, Ma T et al. Antidiabetic drug metformin (GlucophageR) increases biogenesis of Alzheimer's amyloid peptides via up-regulating BACE1 transcription. Proc Natl Acad Sci USA 2009; 106: 3907-3912.

24. Kim HS, Hwang JT, Yun H, Chi SG, Lee SJ, Kang I et al. Inhibition of AMP-activated protein kinase sensitizes cancer cells to cisplatin-induced apoptosis via hyper-induction of p53. J Biol Chem 2008; 283: 3731-3742.

25. Herceg Z, Wang ZQ. Failure of poly(ADP-ribose) polymerase cleavage by caspases leads to induction of necrosis and enhanced apoptosis. Mol Cell Biol 1999; 19: 5124-5133.

26. Moubarak RS, Yuste VJ, Artus C, Bouharrour A, Greer PA, Menissier-de Murcia J et al. Sequential activation of poly(ADP-ribose) polymerase 1, calpains, and Bax is essential in apoptosis-inducing factor-mediated programmed necrosis. Mol Cell Biol 2007; 27: 4844-4862.

27. Wang H, Shimoji M, Yu SW, Dawson TM, Dawson VL. Apoptosis inducing factor and PARP-mediated injury in the MPTP mouse model of Parkinson's disease. Ann N Y Acad Sci 2003; 991: 132-139.

28. Yu SW, Wang H, Poitras MF, Coombs C, Bowers WJ, Federoff HJ et al. Mediation of poly(ADP-ribose) polymerase-1-dependent cell death by apoptosis-inducing factor. Science 2002; 297: 259-263

29. Xu Y, Huang S, Liu ZG, Han J. Poly(ADP-ribose) polymerase-1 signaling to mitochondria in necrotic cell death requires RIP1/TRAF2-mediated JNK1 activation. J Biol Chem 2006; 281: 8788-8795.

30. Mattson MP, Pedersen WA, Duan W, Culmsee C, Camandola S. Cellular and molecular mechanisms underlying perturbed energy metabolism and neuronal degeneration in Alzheimer's and Parkinson's diseases. Ann N Y Acad Sci 1999; 893: 154-175.

31. Wellstead P, Cloutier M. An energy systems approach to Parkinson's disease. Wiley Interdiscip Rev Syst Biol Med 2011; 3: 1-6.

32. Sugiura $Y$, Taguchi R, Setou M. Visualization of spatiotemporal energy dynamics of hippocampal neurons by mass spectrometry during a kainate-induced seizure. PLoS One 2011; 6: e17952.

33. Hardie DG. Minireview: the AMP-activated protein kinase cascade: the key sensor of cellular energy status. Endocrinology 2003; 144: 5179-5183.

34. Suter M, Riek U, Tuerk R, Schlattner U, Wallimann T, Neumann D. Dissecting the role of 5'AMP for allosteric stimulation, activation, and deactivation of AMP-activated protein kinase. J Biol Chem 2006; 281: 32207-32216.

35. Poels J, Spasic MR, Callaerts P, Norga KK. Expanding roles for AMP-activated protein kinase in neuronal survival and autophagy. Bioessays 2009; 31: 944-952.

36. Ronnett GV, Ramamurthy S, Kleman AM, Landree LE, Aja S. AMPK in the brain: its roles in energy balance and neuroprotection. J Neurochem 2009; 109: 17-23.

37. Bolster DR, Crozier SJ, Kimball SR, Jefferson LS. AMP-activated protein kinase suppresses protein synthesis in rat skeletal muscle through down-regulated mammalian target of rapamycin (mTOR) signaling. J Biol Chem 2002; 277: 23977-23980.

38. Chan AY, Dyck JR. Activation of AMP-activated protein kinase (AMPK) inhibits protein synthesis: a potential strategy to prevent the development of cardiac hypertrophy. Can J Physiol Pharmacol 2005; 83: 24-28.

39. Reiter AK, Bolster DR, Crozier SJ, Kimball SR, Jefferson LS. Repression of protein synthesis and mTOR signaling in rat liver mediated by the AMPK activator aminoimidazole carboxamide ribonucleoside. Am J Physiol Endocrinol Metab 2005; 288: E980-E988.

40. Gwinn DM, Shackelford DB, Egan DF, Mihaylova MM, Mery A, Vasquez DS et al. AMPK phosphorylation of raptor mediates a metabolic checkpoint. Mol Cell 2008; 30: 214-226. 
41. Kim SR, Chen X, Oo TF, Kareva T, Yarygina O, Wang $C$ et al. Dopaminergic pathway reconstruction by Akt/Rheb-induced axon regeneration. Ann Neurol 2011; 70: 110-120.

42. Kim SR, Kareva T, Yarygina O, Kholodilov N, Burke RE. AAV transduction of dopamine neurons with constitutively active Rheb protects from neurodegeneration and mediates axon regrowth. Mol Ther 2012; 20: 275-286.

43. Meijer AJ, Codogno P. AMP-activated protein kinase and autophagy. Autophagy 2007; 3 : 238-240.

44. Meley D, Bauvy C, Houben-Weerts JH, Dubbelhuis PF, Helmond MT, Codogno $\mathrm{P}$ et al. AMP-activated protein kinase and the regulation of autophagic proteolysis. $\mathrm{J}$ Biol Chem 2006; 281: 34870-34879

45. Viana R, Aguado C, Esteban I, Moreno D, Viollet B, Knecht E et al. Role of AMP-activated protein kinase in autophagy and proteasome function. Biochem Biophys Res Commun 2008; 369: 964-968.

46. Kim J, Kundu M, Viollet B, Guan KL. AMPK and mTOR regulate autophagy through direct phosphorylation of Ulk1. Nat Cell Biol 2011; 13: 132-141.

47. Narendra D, Tanaka A, Suen DF, Youle RJ. Parkin-induced mitophagy in the pathogenesis of Parkinson disease. Autophagy 2009; 5: 706-708.

48. Vives-Bauza C, Zhou C, Huang Y, Cui M, de Vries RL, Kim J et al. PINK1-dependent recruitment of Parkin to mitochondria in mitophagy. Proc Natl Acad Sci USA 2010; 107: 378-383.

49. Concannon CG, Tuffy LP, Weisova P, Bonner HP, Davila D, Bonner C et al. AMP kinase-mediated activation of the $\mathrm{BH}$-only protein Bim couples energy depletion to stress-induced apoptosis. J Cell Biol 2010; 189: 83-94.

50. Du H, Wolf J, Schafer B, Moldoveanu T, Chipuk JE, Kuwana T. BH3 domains other than Bim and Bid can directly activate Bax/Bak. J Biol Chem 2011; 286: 491-501.

51. Chou SY, Lee YC, Chen HM, Chiang MC, Lai HL, Chang HH et al. CGS21680 attenuates symptoms of Huntington's disease in a transgenic mouse model. J Neurochem 2005; 93 : 310-320.

52. McCullough LD, Zeng Z, Li H, Landree LE, McFadden J, Ronnett GV. Pharmacological inhibition of AMP-activated protein kinase provides neuroprotection in stroke. J Biol Chem 2005; 280: 20493-20502.
53. Lopez-Lopez C, Dietrich MO, Metzger F, Loetscher H, Torres-Aleman I. Disturbed cross talk between insulin-like growth factor I and AMP-activated protein kinase as a possible cause of vascular dysfunction in the amyloid precursor protein/presenilin 2 mouse model of Alzheimer's disease. J Neurosci 2007; 27: 824-831.

54. Ju TC, Chen HM, Lin JT, Chang CP, Chang WC, Kang JJ et al. Nuclear translocation of AMPK-alpha1 potentiates striatal neurodegeneration in Huntington's disease. J Cell Biol 2011; 194: 209-227.

55. Mairet-Coello G, Courchet J, Pieraut S, Courchet V, Maximov A, Polleux F. The CAMKK2AMPK Kinase Pathway Mediates the Synaptotoxic Effects of Abeta Oligomers through Tau Phosphorylation. Neuron 2013; 78: 94-108.

56. Hagberg H, Wilson MA, Matsushita H, Zhu C, Lange M, Gustavsson M et al. PARP-1 gene disruption in mice preferentially protects males from perinatal brain injury. $J$ Neurochem 2004; 90: 1068-1075.

57. Sugiura $Y$, Shimma S, Setou M. Thin Sectioning Improves the Peak Intensity and Signal-to-Noise Ratio in Direct Tissue Mass Spectrometry. J Mass Spectrom Soc Jpn 2006; $54: 4$.

58. Schwartz SA, Reyzer ML, Caprioli RM. Direct tissue analysis using matrix-assisted laser desorption/ionization mass spectrometry: practical aspects of sample preparation. J Mass Spectrom 2003; 38: 699-708.

59. lancu R, Mohapel P, Brundin P, Paul G. Behavioral characterization of unilateral 6-OHDA-lesion model of Parkinson's disease in mice. Behav Brain Res 2005; 162: $1-10$.

60. Mori A, Ohashi S, Nakai M, Moriizumi T, Mitsumoto Y. Neural mechanisms underlying motor dysfunction as detected by the tail suspension test in MPTP-treated C57BL/6 mice. Neurosci Res 2005; 51: 265-274.

(i) (2) Cell Death and Disease is an open-access journal published by Nature Publishing Group. This work is licensed under a Creative Commons Attribution-NonCommercialShareAlike 3.0 Unported License. To view a copy of this license, visit http://creativecommons.org/licenses/by-nc-sa/3.0/ 